\title{
Feasibility of Utilising 3D software in Occupational Therapy Practice
}

\author{
Arthur Money(1), Anita Atwal (2), Georgia Spiliotopoulou@brunel.ac.uk(2), Michele Turko (3), Anand Unadkat (4) \\ (1)Lecturer in the Department of Information Systems and Computing at Brunel University \\ (2)Lecturer in Occupational Therapy, School of Health and Social Care at Brunel University, \\ (3)Pre- Registration Masters Occupational Therapy, School of Health and Social Care at Brunel University \\ (4)Student, BSc (Hons), Department of Information Systems and Computing at Brunel University
}

\begin{abstract}
Arthur.Money@brunel.ac.uk; Anita.Atwal@brunel.ac.uk; Georgia.Spiliotopoulou@brunel.ac.uk 1101426@brunel.ac.uk; unadkat.anand@gmail.com
\end{abstract}

\section{INTRODUCTION}

Occupational home visits for older adults are a standard part of the discharge process within the NHS. However, there is limited research on the efficacy of this practice (Atwal et al 2012) or on alternative approaches, including the use of technology, for carrying out this intervention more effectively. Home visits typically involve a short visit with the patient to their home to assess their ability to perform usual everyday tasks. Consequently, the practitioner proposes recommendations for adaptations to the patient's home and for the provision of specialist assistive equipment such as bed hoists, support rails, shower seats and grab rails and so forth.

Existing home visit practice, however, is far from optimal. Patients have reported that they find the home visit process anxiety-provoking, and feel as if they are being tested/assessed in terms of their mobility around the home and hence do not feel able to collaborate as an equal partner for fear of not being discharged home after the visit (Atwal, McIntyre et al. 2007). Older adults in particular have also perceived the process as demoralising, daunting and increasing their anxiety (Clark and Dyer 2008, Atwal,McIntyre et al 2007 Atwal, Spiliotopoulou et al 2012 . Consequently more than $50 \%$ of home adaptations and equipment installed is not used by the patient once discharged to home which results in sub-optimal health outcomes and wastage of resources (Nygard, Grahn et al. 2000). This is perhaps no surprise when considering how intensely personalised and sensitive the home setting is (Crabtree 2003) coupled with the fact that there is no readily available tool or technique that assists patients and practitioners to jointly visualise and explore the home environment and experiment with the variety of options that are available to adapt the home according to patients' personal needs (Atwal, Money et al. 2013r).

\section{Bringing the home into the hospital}

This research proposes to use digital 3D interior design software (3DIDS) to "bring the home into the hospital". It is hypothesised that working with 3D visual representations of the patient's home could result in better patient/therapist decision making, particularly in relation to home modifications an assistive technology. Existing research proposes that future 3D work in a medical setting would include being able to individualize patient treatment planning and give a visual demonstration that could help inform patient decisions (Schendel, 2000).

3DIDS is one application of virtual reality (VR) technology which primarily allows individuals to design or redesign their homes virtually, prior to going ahead with these changes in the real world. This technology enables occupational therapists to rapidly create the 3D representation of the patient's home and provides an interactive simulation of the home area which can be easily navigated through in real time. Figure 1 is an example of home interior representations developed using 3DIDs software.

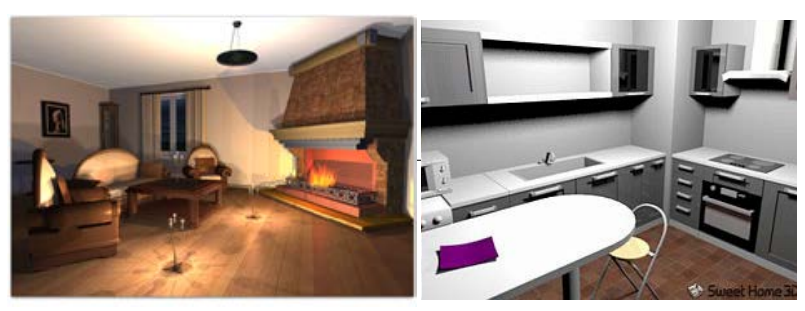

Figure 1: Example of home interior developed using 3DIDS 
This research proposes to explore how the use of 3DIDS could aid the home discharge process and gain insights into patient and practitioner thoughts about its application in practice. The prospect of using VRIDS has potential to respond to a number of the issues that currently limit the effectiveness of the current home discharge process, for example:

- 3DIDS could serve as a tool that enables the patient and practitioner to jointly visualise the interior of the home and trial a range of adaptations and specialist equipment within it.

- Enhance collaboration between clinician and patient and assist them in making shared decisions about how this sensitive and personalised space may be best adapted specifically to the patient's individual needs.

- Provide an interactive simulation of the home enabling the patient to "walk" through the home, via a PC or laptop, to help therapists better consider barriers to everyday performance and enhance the patient's insight and motivation to participate in tailored interventions.

- Provide the patient with the valuable opportunity to consult as an expert on their own needs, and participate as an equal partner in decision making.

If a new technology is to be implemented successfully, then it needs to be developed for, and accepted by the people who will both use and benefit from this (Schaper \& Pervan 2007). As a step towards applying 3DIDS to the occupational therapy home discharge process, a series of focus groups and interactive sessions were conducted with occupational therapists who work within the NHS sector. The results of these focus groups will be used to further develop and customise the functionality of an existing open sourced 3DIDS application.

\section{METHODS}

The research carried out to date has been executed in two key phases:

1) A pilot study, via a series of focus groups, to explore the 3DIDS application feasibility, from an occupational therapists' (OT) perspective and to identify initial design requirements.

2) Implement the design requirements in the form of a working prototype and ascertain OT perceptions of the ease of use of application via a series of interactive sessions.

\section{RESULTS}

Phase one: The results revealed that occupational therapists in general had a positive attitude towards the potential value of this software within the homedischarge process. Therapists perceived that the software had a number of benefits: 1) in improving the multi-agency decision making process; 2) in improving levels of patient engagement and efficiency by making better use of less senior staff's time. Still, a number of refinements were suggested to better suit the needs of the occupational therapists and the clients.

Phase two: A plugin to an open sourced off-theshelf 3DIDS application (SweetHome 3D) was developed to take steps towards tailoring the functionality of the 3DIDS software to OT needs. The findings from trialling the customised application with OT's in a series of think aloud sessions are as follows: Negative perceptions; 1 ) The technology felt outdated; 2) Controls are good but quite tricky to operate; 3 ) Mouse too sensitive at times. Positive Perceptions; 1) Easy to use, 2) Rotation of person good, 3) good colours. Personalisation: 1) Need for personalised objects specific to patient in object library. Potential Clinical Utility: 1) Potential use within access visits to show patients where modifications would be placed and assist in decision making, 2) When major adaptations are made to a patient's home, it would assist with reading of plans and decision making, 3) Useful for patients who are difficult to engage with.

\section{FUTURE WORK}

Phase three of this research will involve a series of focus groups with OT's that have used the customised 3DIDS software application. There are a number of questions that still need to be answered in order to explore the feasibility and clinical context in which this software may be applied, for example:

Will users be able to relate it to their real world? Would one object that has personal meaning for them e.g. a 3D representation of their pet within the home environment facilitate this process and bring it to life? Developers are faced with challenges of building not only a robust application, but also taking into accounts the usability factors of the systems which are unique for each rehabilitation scenario. Should the research focus be on piloting the 3D technology for one specific client group, or with the aim of engaging patients across a range of demographic groups and abilities?

The findings from our research suggest that OTs view the $3 \mathrm{D}$ technology as enhancing practice. Thus they may be willing to adopt it if they view the software as usable. Thus we need to ensure that the software meets their working requirements in order to enhance quality of care for patients and careers.

\section{REFERENCES}

Atwal, A. McIntyre A et al. (2007). "Older adults and carers' perceptions of pre-discharge occupational therapy home visits in acute care." Age and Ageing 37(1): 72-76. 
Atwal A; Spiliotopoulou G.et al. (2012) Older adults' experiences of occupational therapy predischarge home visits: a systematic thematic synthesis of qualitative research. British Journal of Occupational Therapy 75 (3),118-127.

Atwal, A. Money G et al. (2013). "Occupational therapists' perceptions about the clinical utility of the 3D interior design software." Disability and Rehabilitation: Assistive Technology (4):348-55.

Clark H , Dyer S (1998) Equipped for going home from hospital. Health Care in Later Life (3), 1, 3545

Crabtree, A., Hemmings, T., Rodden, T.,

Chevherst, K., Clarke, K., Dewsbury, G., Hughes, J. and Rouncefield, M. (2003). Designing with care: adapting cultural probes to inform design in sensitive settings. $\mathrm{OzCHI}$. Brisbane, Australia, UQ Print: 4-13.
Nygard, N., Grahn U et al. (2000). "Reflecting on practice: are home visits prior to discharge worthwhile?" Scandinavian Journal of Caring Science 18(210;183-203.

Schaper LK, Pervan GP (2007) ICT and OTs: A model of information and communication technology acceptance and utilisation by occupational therapists. International Journal of Medical Informatics 76: S212-21.

Schendel, S.A. (2000) "Three-dimensional surgical simulation in resident training", Aesthetic Surgery Journal 20(3 260-261. 